\title{
High expression of prostate-specific membrane antigen in the tumor-associated neo- vasculature is associated with worse prognosis in squamous cell carcinoma of the oral cavity
}

Michael C Haffner ${ }^{1,2, *}$, Johannes Laimer ${ }^{1, *}$, Alcides Chaux ${ }^{3}$, Georg Schäfer ${ }^{4,5}$, Peter Obrist ${ }^{4}$, Andrea Brunner ${ }^{4}$, Irmgard E Kronberger ${ }^{6}$, Klaus Laimer ${ }^{7}$, Bora Gurel ${ }^{3}$, Johann-Benedikt Koller $^{1}$, Christof Seifarth ${ }^{5}$, Bettina Zelger ${ }^{4}$, Helmut Klocker ${ }^{5}$, Michael Rasse ${ }^{7}$, Wolfgang Doppler ${ }^{1}$ and Neil H Bander ${ }^{2}$

${ }^{1}$ Division of Medical Biochemistry, Biocenter, Innsbruck Medical University, Innsbruck, Austria; ${ }^{2}$ Department of Urology, Weill Medical College of Cornell University, New York, NY, USA; ${ }^{3}$ Department of Pathology, Johns Hopkins University, Baltimore, MD, USA; ${ }^{4}$ Department of Pathology, Innsbruck Medical University, Innsbruck, Austria; ${ }^{5}$ Department of Urology, Innsbruck Medical University, Innsbruck, Austria; ${ }^{6}$ Department of General and Transplant Surgery, Innsbruck Medical University, Innsbruck, Austria and ${ }^{7}$ Division of Maxillofacial Surgery, Innsbruck Medical University, Innsbruck, Austria

Prostate-specific membrane antigen (PSMA) is a transmembrane protein expressed in prostate cancer as well as in the neo-vasculature of nonprostatic solid tumors. Here, we determined the expression pattern of PSMA in the vasculature of oral squamous cell carcinoma. Using a previously validated antibody, PSMA staining distribution and cyclooxygenase 2 (COX2) expression status was evaluated in a cohort of patients with squamous cell carcinoma of the oral cavity $(n=96)$ using immunohistochemistry and was correlated with clinicopathological features as well as outcome. Twenty-four $(25 \%)$ cases showed no detectable PSMA staining, $48(50 \%)$ demonstrated positive immunoreactivity for PSMA in less than $50 \%$ of microvessels and $24(25 \%)$ cases showed strong endothelial PSMA expression in more than $\mathbf{5 0} \%$ of tumor-associated microvessels. High endothelial PSMA expression was associated with greatly reduced survival (18.2 vs 77.3 months; $P=0.0001$ ) and maintained prognostic significance after adjusting for grade and stage in multivariate analysis (hazard ratio $=2.19, P=0.007$ ). Furthermore, we observed a strong association between endothelial PSMA and cancer cell-specific COX2 expression. In conclusion, we provide the first evidence for the prognostic significance of endothelial PSMA expression in oral squamous cell carcinoma and, suggest a potential interaction between arachidonic acid metabolites and endothelial PSMA expression in the tumor neo-vasculature.

Modern Pathology (2012) 25, 1079-1085; doi:10.1038/modpathol.2012.66; published online 30 March 2012

Keywords: COX2; FOLH1; oral squamous cell carcinoma; prognosis; prostate-specific membrane antigen; PSMA; vasculature

The formation of new blood vessels in the tumor microenvironment represents a crucial step in the progression of many solid tumors. ${ }^{1,2}$ Several ther-

Correspondence: Dr MC Haffner, MD, Division of Medical Biochemistry, Biocenter, Innsbruck Medical University, Fritz Pregl Street 3, A-6020 Innsbruck, Austria.

E-mail: michael.c.haffner@gmail.com

*These authors contributed equally to this work.

Received 26 January 2012; revised 1 March 2012; accepted 1 March 2012; published online 30 March 2012 apeutic approaches have therefore focused on inhibiting neo-angiogenesis and have shown sustainable clinical responses. ${ }^{2}$ Such anti-angiogenic therapies can either interfere with signal transduction pathways or signaling molecules that regulate vessel formation and vessel growth or directly target tumor-associated endothelial cells. ${ }^{3}$ It is of particular interest that several markers have been characterized that show a specific expression in tumor-associated endothelial cells, but not in normal endothelial cells. ${ }^{2,4,5}$ One such molecule, that 
shows high expression in the tumor-associated neovasculature is prostate-specific membrane antigen (PSMA, also known as folate hydrolase 1, FOLH1). PSMA is a metallo-protease that was originally cloned from human prostate carcinoma cells and has been shown to be overexpressed in the vast majority of prostate cancers. ${ }^{6,7}$ Subsequently several studies suggested that PSMA expression is not restricted to prostate epithelial cells but can also be found in tumor-associated endothelial cells in many different solid malignancies. ${ }^{8-12}$ The functional role of endothelial PSMA is not known and, as recent studies demonstrated, endothelial PSMA expression can vary significantly within and between different tumor types. ${ }^{12-14}$

Cyclooxygenase 2 (COX2) is a key enzyme in the arachidonic acid metabolism involved in the biosynthesis of prostagladins, which contribute to a pro-angiogenic microenvironment in tumors. ${ }^{15}$ Expression of COX2 is upregulated in squamous cell carcinoma and treatment with COX2 selective inhibitors results in reduced tumor growth. ${ }^{16,17}$ Furthermore, a large body of evidence demonstrates that inhibition of COX2 enzymatic reaction results in substantial changes in the tumor-associated neo-vasculature, suggesting that COX2 could have a key role in regulating tumor-associated neoangiogenesis. ${ }^{18-20}$

To further elucidate the role of PSMA in oral squamous cell carcinoma, we evaluated the expression pattern of PSMA in a series of oral squamous cell carcinoma by immunohistochemistry and correlated PSMA levels with clinicopathological features as well as clinical outcome data. We show that PSMA is detectable in a subset of lesions and that endothelial PSMA expression correlates with expression of COX2 in cancer cells. Furthermore, we provide first evidence that high endothelial PSMA expression is associated with worse prognosis.

\section{Materials and methods}

\section{Patients}

In this retrospective study, 96 patients who underwent surgery at the Department of Maxillofacial Surgery, Innsbruck Medical University, between 1980 and 1997 and for whom tissue specimens and clinical follow-up data were available were included. Paraffin-embedded tissues from the initial surgical specimens were retrieved and tissue micro arrays (TMAs) containing representative oral squamous cell carcinomas lesions from each patient were constructed as described previously. ${ }^{21}$ Patients were followed-up from the date of surgery with a mean follow-up period of 48 months (range, 1-245 months). Clinical and pathological characteristics of the study population are summarized in Table 1.
Table 1 Clinicopathological features of 96 patients with oral squamous cell carcinoma

\begin{tabular}{lc}
$\begin{array}{l}\text { Clinicopathological } \\
\text { features }\end{array}$ & $\begin{array}{c}\text { No. of Cases } \\
(\%)\end{array}$ \\
\hline $\begin{array}{l}\text { Gender } \\
\text { Male }\end{array}$ & $74(77)$ \\
Female & $22(23)$ \\
Age, years & \\
Mean & 64 \\
Range & $26-85$ \\
Clinical stage & \\
I & $5(5)$ \\
II & $6(6)$ \\
III & $24(25)$ \\
IV & $61(64)$ \\
Histological grade & \\
Grade 1 & $18(19)$ \\
Grade 2 & $53(55)$ \\
Grade 3 & $25(26)$ \\
Tumor size & \\
< 2 cm & \\
2-4 cm & $6(6)$ \\
> cm & $24(25)$ \\
Not specified & $32(33)$ \\
Adjuvant therapy & $34(36)$ \\
Chemotherapy & \\
Radiotherapy & \\
\hline
\end{tabular}

\section{Immunohistochemistry}

Four to six micron thick sections were mounted on glass slides, deparaffinized by Histo-Clear (National Diagnostics, Atlanta, GA, USA) and rehydrated. Immunostaining of PSMA and CD31 was performed as described previously. ${ }^{12}$ Briefly, slides were either pressure cooked for $1 \mathrm{~min}$ in $10 \mathrm{mM}$ citrate buffer $\mathrm{pH}$ 6.0 for CD31 staining or incubated in Target Retrieval Solution pH 9.0 (cat no. S2368; Dako, Carpinteria, CA, USA) at $95^{\circ} \mathrm{C}$ for $30 \mathrm{~min}$ for PSMA staining. Endogenous peroxidase was blocked with Peroxidase Block (cat no. K4006; Dako) for $5 \mathrm{~min}$. Sections of the same TMA block were then incubated with either anti-CD31 (clone 1A10; Novocastra - Vision BioSystems, Norwell, MA, USA) or antiPSMA antibody (clone 3E6; Dako) diluted 1:25 and 1:20, respectively, in Antibody Diluent (cat no. S0809; Dako) for $60 \mathrm{~min}$. Immunohistochemical detection of COX2 was performed using a murine monoclonal COX2-specific antibody (Cayman, USA) as described previously. ${ }^{22}$ Briefly, deparaffinized tissue sections were incubated for $15 \mathrm{~min}$ at $90{ }^{\circ} \mathrm{C}$ and the primary antibody was applied at 1:100 dilution for $2 \mathrm{~h}$ at room temperature. Slides were then further treated with anti-mouse labeled polymer HRP for $60 \mathrm{~min}$ followed by $8 \mathrm{~min}$ in diaminobenzidine solution (Envision System HRP, cat no. K4006; Dako). Finally, all slides were counterstained with Harries-modified Hematoxylin (Fisher Scientific, Pittsburgh, PA, USA). 


\section{Evaluation of Slides}

To determine microvessel density (MVD), CD31positive vascular structures were evaluated for each TMA spot per high power field and categorized in low MVD (less than 10 CD31-positive structures), intermediate MVD (11-25 CD31-positive structures) and high MVD (more than 26 CD31-positive structures).

PSMA expression in the tumor vasculature was scored semiquantitatively. Lesions with a 0-staining score showed no detectable endothelial PSMA expression; lesions with a staining score of 1 showed detectable PSMA staining in 5-50\% of microvessels; a staining score of 2 was given for lesions that showed positive PSMA staining in more than 50\% of tumor-associated microvessels. For survival analysis lesions with a PSMA-staining score of 0 and 1 were grouped (low PSMA) and compared with lesions with a staining score 2 (high PSMA).

COX2 staining was evaluated by determining the percentage of cancer cells that showed positive staining. Lesions with more than $70 \%$ of cancer cells showing positive COX2 staining were classified as high COX2 expressing.

\section{Statistical Methods}

Categorical variables were compared using Fisher's exact test. Effect size was estimated using Cramér's $V$ coefficient. Survival curves were generated using the Kaplan-Meier method and compared using the log-rank (Mantel-Cox) test. Hazard ratios (HR) with their corresponding 95\% confidence intervals (CI) were estimated using Cox proportional hazards models. Multivariate adjusted for tumor grade, clinical stage and endothelial PSMA or tumor COX2-staining status. For categorical variables, an association was considered as nonrandom if twotailed $P<0.01$ and strong if Cramér's $V>0.30$. HRs were interpreted using 95\% CI. Data were analyzed using Stata/SE release 11.2 (StataCorp LP, College Station, TX, USA).

\section{Results}

To evaluate the expression pattern of PSMA in oral squamous cell carcinoma we used a previously validated antibody and stained a well-annotated cohort of oral squamous cell carcinoma samples. ${ }^{12,21,23}$ All clinicopathological feature of the patient cohort are summarized in Table 1. A total of 96 individual samples were investigated. Twenty four (25\%) samples showed no detectable PSMA staining, $48(50 \%)$ demonstrated positive immunoreactivity for PSMA in a subset of tumorassociated microvessels and 24 (25\%) showed strong endothelial PSMA expression in the majority of vascular structures (Figure 1a). Additionally, MVD was assessed by staining for CD31 with 13 (13\%) patients showing low, 45 (47\%) intermediate and $38(40 \%)$ high MVD (Figures $1 \mathrm{~b}$ and d). Interestingly, PSMA expression was strongly associated with MVD $(P=0.002, V=0.34)$. We also noted a significant difference in overall survival between patients with low vs high endothelial PSMA expression (77 vs 18 months; $P=0.0001$, Table 2, Figure 3a). After adjusting for grade and stage, high PSMA expression remained an independent marker for poor prognosis $(\mathrm{HR}=2.19$, 95\% CI 1.2-3.9, $P=0.007$, Table 3).

Furthermore, we assessed COX2 protein expression levels by immunohistochemistry. The majority of lesions showed detectable cytoplasmic COX2 staining in at least a subset of cancer cells (Figure 2). Twenty-seven cases (28\%) exhibited strong staining in the majority $(>70 \%)$ of cells. High COX2 expression was associated with shorter overall survival (74 vs 32 months; $P=0.007$, Table 2, Figure $3 \mathrm{~b}$ ), which remained statistically significant in a multivariate analysis after adjusting for grade and stage $(\mathrm{HR}=1.81,95 \%$ CI 1.1-3.0, $P=0.02$, Table 3$)$. COX2 expression was not associated with MVD. Interestingly, however, we observed a strong association between COX2 and PSMA expression $(P=0.003, V=0.33)$ and lesions with high endothelial PSMA levels tended to show strong COX2 staining in the cancer cells suggesting a possible link between COX2-derived prostaglandins and endothelial PSMA expression.

\section{Discussion}

Tumor growth crucially depends on neo-vascularization. ${ }^{1,2}$ As a result, selectively targeting and inhibition of neo-angiogenesis may be an effective approach to treat solid tumors. ${ }^{2,3}$ PSMA has been suggested as a potential candidate for direct tumorspecific vascular targeting. ${ }^{2}$ Interestingly, neo-vascular PSMA expression levels appear to differ significantly between different tumor types. ${ }^{9-12,24,25}$ Here, we show that in oral squamous cell carcinoma high neo-vascular PSMA expression can only be detected in a subset of lesions. This finding is somewhat in contrast to previously published data from other solid malignancies in which the majority of tumors showed strong endothelial PSMA expression and suggests that differences in the tumor microenvironment may influence the differential expression of PSMA in the tumor-associated neovasculature. ${ }^{8,12,26,27}$

The function of PSMA in endothelial cell biology is not well established. Insights from in vitro and in vivo model systems suggest that PSMA could contribute to endothelial cell invasion through its peptidase function. ${ }^{28}$ Indeed, it was shown that endothelial cells lacking PSMA show less efficient matrix invasion. This phenotype was suggested to reflect the function of PSMA in a regulatory loop involving b1 integrin and PAK1, which mediate neoangiogenesis through cell invasion. ${ }^{28}$ Furthermore, 

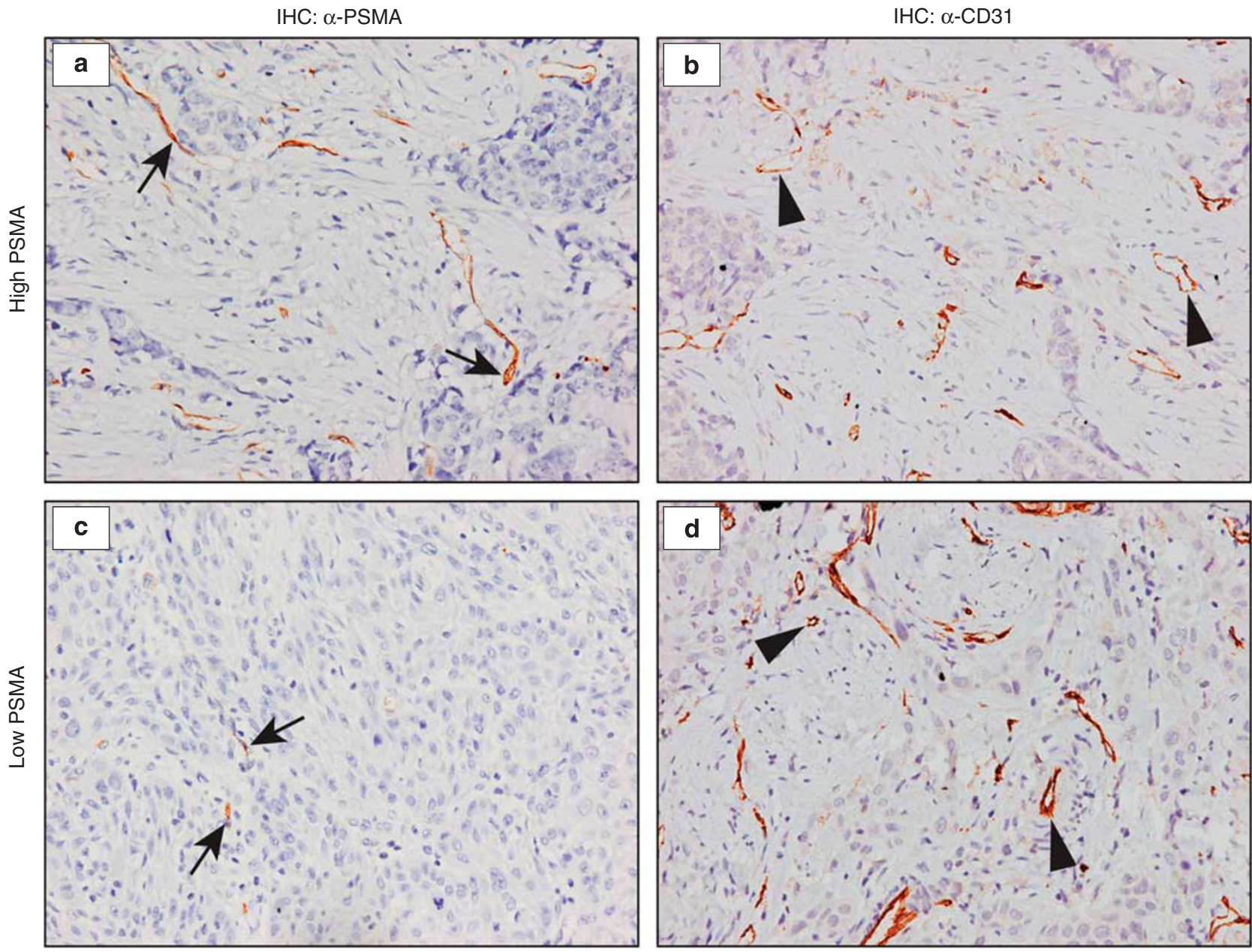

Figure 1 Prostate-specific membrane antigen (PSMA) is detectable in the tumor-associated microvasculature of squamous cell carcinoma of the oral cavity. (a) Representative micrograph of endothelial PSMA staining with staining score 2 (high PSMA expression). (c) Representative micrograph of tumors with PSMA staining score 1 (low PSMA expression). Arrows indicate PSMA-positive micro vessels. (b, d) Corresponding CD31 staining, arrowheads indicate micro-vascular structures.

Table 2 Estimates for 5-year and 10-year survival rates in 96 patients with oral squamous cell carcinoma

\begin{tabular}{lcc}
\hline & $\begin{array}{c}5 \text {-year } \\
\text { survival, } \% \\
(95 \% \text { CI })\end{array}$ & $\begin{array}{c}10 \text {-year } \\
\text { survival, } \% \\
(95 \% \text { CI })\end{array}$ \\
\hline Overall survival & $34(25,44)$ & $18(10,28)$ \\
Histological grade & & \\
Grade 1 & $72(46,87)$ & $37(11,63)$ \\
Grade 2 & $31(19,44)$ & $13(5,25)$ \\
Grade 3 & $16(5,33)$ & $16(5,33)$ \\
PSMA expression & & $23(13,36)$ \\
Low PSMA & $43(32,55)$ & \\
High PSMA & $6(1,23)$ & $24(13,36)$ \\
COX2 expression & & $4(0,17)$ \\
Low COX2 & $39(27,50)$ & \\
High COX2 & $20(7,37)$ &
\end{tabular}

Abbreviations: CI, confidence interval; COX2, cyclooxygenase 2; PSMA, prostate-specific membrane antigen.

${ }^{\mathrm{a}}$ No patients survived more than 10 years in this category.
Table 3 Cox proportional hazards model for prostate-specific membrane antigen (PSMA) and cyclooxygenase 2 (COX2) expressions in 96 patients with oral squamous cell carcinoma

Adjusted hazard ratio ${ }^{\mathrm{a}}(95 \% \mathrm{CI})$

\section{PSMA expression \\ Low PSMA ${ }^{\mathrm{b}}$ \\ High PSMA \\ COX2 expression \\ Low COX2 $2^{\mathrm{b}}$ \\ High COX2}

1

$2.19(1.24,3.89)$

1

$1.79(1.08,2.96)$

Abbreviations: CI, confidence interval; COX2, cyclooxygenase 2; PSMA, prostate-specific membrane antigen.

${ }^{a}$ Adjusted for clinical stage and histological grade.

${ }^{\mathrm{b}}$ Reference category.

through its role in folate metabolism PSMA could also have an important function in the regulation of folate homeostasis and Gordon et $a l^{23}$ recently suggested that the PSMA-mediated folate uptake 
High COX2

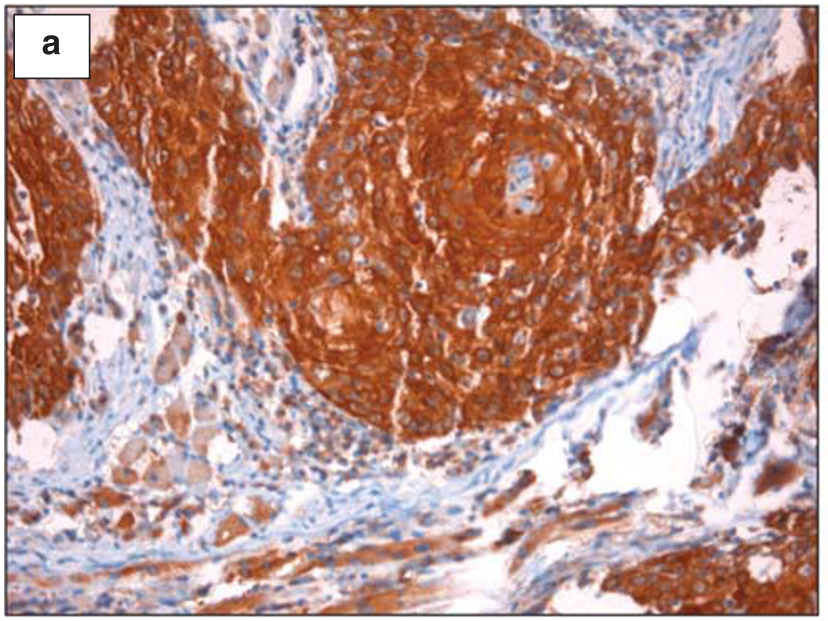

Low COX2

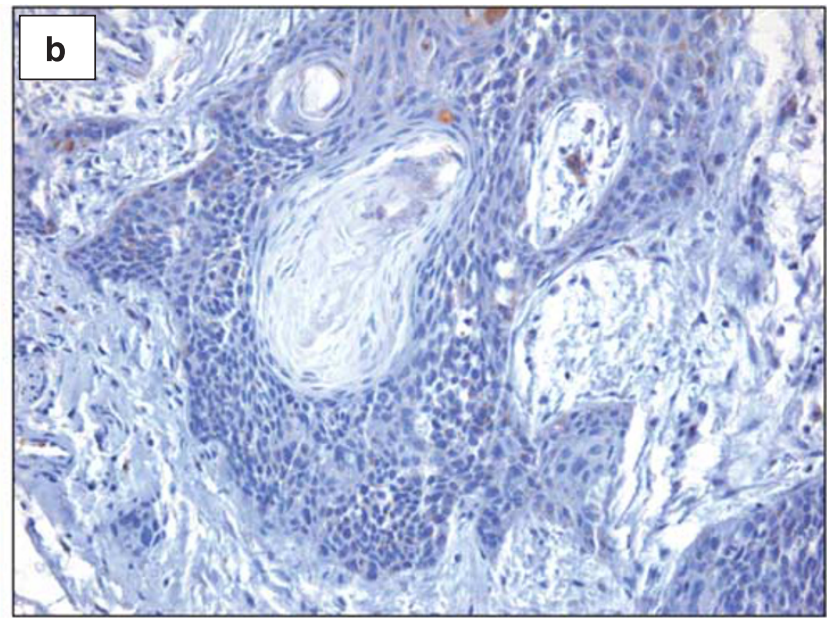

Figure 2 (a) Representative micrograph of lesion with high cyclooxygenase 2 (COX2) expression. (b) Representative micrograph of COX2-negative lesion.

could be required to regenerate essential cofactors of endothelial nitric oxide synthase.

PSMA expression in prostate cancer cells is regulated by the androgen receptor and potentially other oncogenic transcription factors. ${ }^{29,30}$ The transcriptional regulation of PSMA in endothelial cells, however, is not well characterized. Recent experimental evidence suggests that soluble factors, secreted by cancer cells could control endothelial PSMA expression. For instance, conditioned medium from cancer cell lines can increases PSMA expression in human umbilical vein endothelial cells in vitro. ${ }^{31}$ This finding raises the question of the nature of factors involved in PSMA transcriptional regulation. In general, angiogenesis is a complex mechanism that involves a plethora of signaling molecules. ${ }^{32,33}$ Prostaglandins, which are generated through the conversion of arachidonic acid, a process that involves cyclooxygenases as a limiting step have been suggested to have a key role in tumor-associated neo-angiogenesis. ${ }^{34}$ High levels of COX2-derived prostaglandin $\mathrm{E}(2)$ for instance have been described in highly vascularized tumors and selective inhibition of COX2 results in decreased tumor growth because of the changes in the neo-vasculature. ${ }^{18-20,34}$ Interestingly, we observed a strong association between epithelial COX2 expression and neo-vascular PSMA expression and lesions with high epithelial COX2 expression tended to have high endothelial PSMA expression. This finding suggests that COX2derived arachidonic acid metabolites could be involved in the regulation of endothelial PSMA expression. Although strictly correlative at this point, the strong association between COX2 and PSMA certainly warrants further mechanistic evaluation.

Furthermore, we demonstrate for the first time that PSMA expression in the neo-vasculature of oral squamous cell carcinoma is a strong prognostic marker. Patients with high PSMA expression showed a dramatically decreased survival as compared to patients with low endothelial PSMA staining (Table 2, Figure 3) and the prognostic value of PSMA was maintained in a multivariate analysis after adjusting for grade and stage (Table 3). It is worth noting that high expression of PSMA in the neovasculature has recently also been shown to have prognostic significance in osteosarcoma, suggesting that at least in a subset of solid tumors, endothelial PSMA expression could promote a more aggressive phenotype. ${ }^{25}$ The robust immunohistochemical staining combined with the straight-forward scoring could allow the routine clinical evaluation of this marker and would eventually help to identify patients with highly aggressive disease. Future prospective studies in larger patient cohorts are therefore necessary to further evaluate the prognostic and also clinical significance of PSMA in squamous cell carcinoma of the oral cavity.

Two clinical trials have recently established the feasibility of vascular targeting using PSMA-specific antibodies $^{35,36}$ and a number of PSMA-targeting molecules ranging from monoclonal antibodies to PSMA-binding ligands, RNA aptamers, targeted nanoparticles and viral PSMA-targeting systems are currently under pre-clinical or clinical investigation in different tumor types. ${ }^{37-41}$ Given the strong association of PSMA expression with survival it remains to be shown if a PSMA-specific targeting approach would show a therapeutic benefit in a subset of patients with oral squamous cell carcinoma.

In conclusion, we provide first evidence for the neo-vascular expression of PSMA in squamous cell carcinoma of the oral cavity and show that PSMA is a strong prognostic marker in oral squamous cell carcinoma. Furthermore, we demonstrate an association between COX2 expression and endothelial PSMA levels, which could indicate a potential mechanistic interaction between prostaglandin signaling and neo-vascular PSMA expression. 

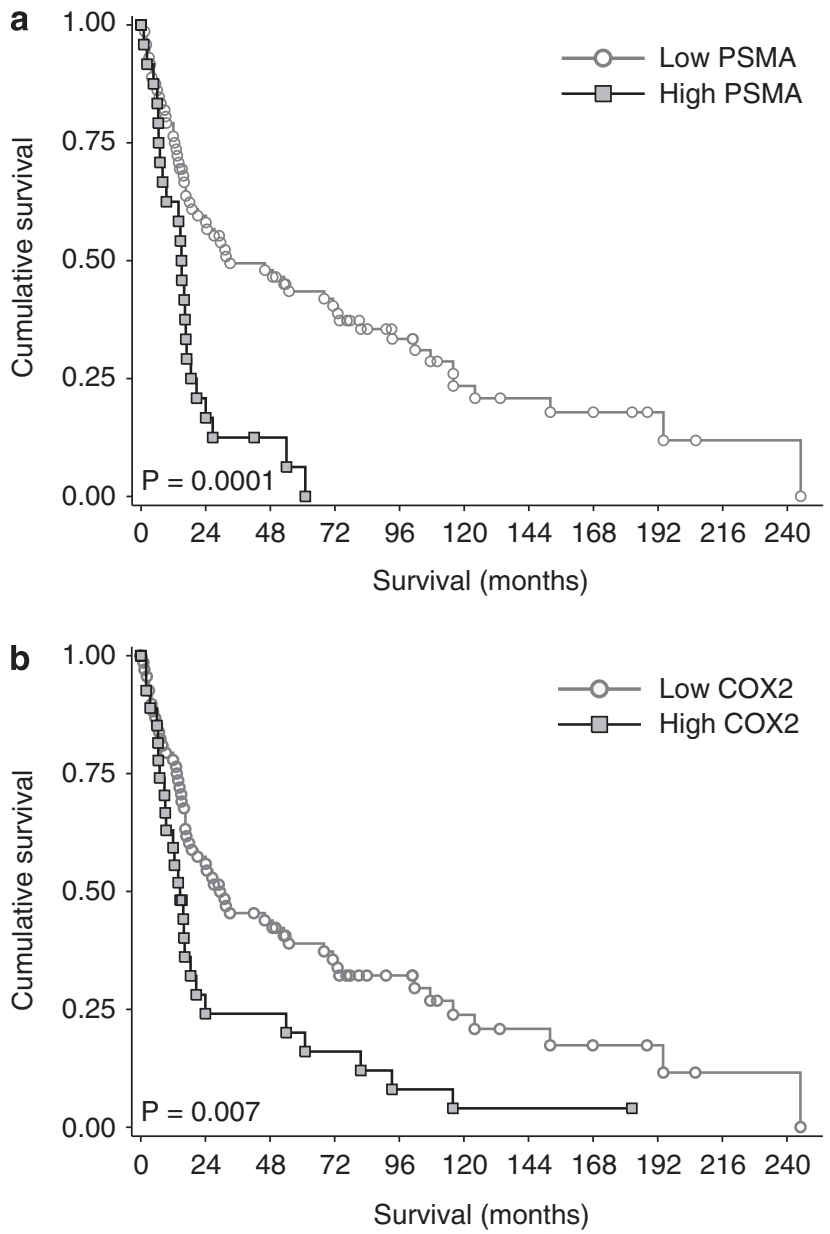

Figure 3 (a) Kaplan-Meier survival curves showing cumulative overall survival for patients with low (staining score $=0$ and 1) and high endothelial Prostate-specific membrane antigen (PSMA) staining (staining score=2). (b) Kaplan-Meier survival curves showing cumulative overall survival for patients with high ( $>70 \%$ cancer cells positive) and low $(<70 \%$ of cancer cells positive) cyclooxygenase 2 (COX2) expression.

\section{Acknowledgement}

The authors want to thank Peggy Moy for technical assistance, Dr He Liu and Dr Angelo DeMarzo for stimulating discussions and Lana Winter for administrative assistance.

\section{Disclosure/conflict of interest}

The authors declare no conflict of interest.

\section{References}

1 Folkman J. Role of angiogenesis in tumor growth and metastasis. Semin Oncol 2002;29:15-18.

2 Neri D, Bicknell R. Tumour vascular targeting. Nat Rev Cancer 2005;5:436-446.

3 Folkman J. Angiogenesis: an organizing principle for drug discovery? Nat Rev Drug Discov 2007;6:273-286.
4 Seaman S, Stevens J, Yang MY, et al. Genes that distinguish physiological and pathological angiogenesis. Cancer Cell 2007;11:539-554.

5 St Croix B, Rago C, Velculescu V, et al. Genes expressed in human tumor endothelium. Science 2000;289: 1197-1202.

6 Ross JS, Sheehan CE, Fisher HA, et al. Correlation of primary tumor prostate-specific membrane antigen expression with disease recurrence in prostate cancer. Clin Cancer Res 2003;9:6357-6362.

7 Chang SS, Gaudin PB, Reuter VE, et al. Prostatespecific Membrane Antigen: Much more than a Prostate Cancer Marker. Mol Urol 1999;3:313-320.

8 Chang SS, O'Keefe DS, Bacich DJ, et al. Prostatespecific membrane antigen is produced in tumorassociated neovasculature. Clin Cancer Res 1999;5: 2674-2681.

9 Chang SS, Reuter VE, Heston WD, et al. Five different anti-prostate-specific membrane antigen (PSMA) antibodies confirm PSMA expression in tumor-associated neovasculature. Cancer Res 1999;59:3192-3198.

10 Liu H, Moy P, Kim S, et al. Monoclonal antibodies to the extracellular domain of prostate-specific membrane antigen also react with tumor vascular endothelium. Cancer Res 1997;57:3629-3634.

11 Silver DA, Pellicer I, Fair WR, et al. Prostate-specific membrane antigen expression in normal and malignant human tissues. Clin Cancer Res 1997;3:81-85.

12 Haffner MC, Kronberger IE, Ross JS, et al. Prostatespecific membrane antigen expression in the neovasculature of gastric and colorectal cancers. Hum Pathol 2009;40:1754-1761.

13 Baccala A, Sercia L, Li J, et al. Expression of prostatespecific membrane antigen in tumor-associated neovasculature of renal neoplasms. Urology 2007;70:385-390.

14 Samplaski MK, Heston W, Elson P, et al. Folate hydrolase (prostate-specific antigen) 1 expression in bladder cancer subtypes and associated tumor neovasculature. Mod Pathol 2011;24:1521-1529.

15 Furstenberger G, Krieg P, Muller-Decker K, et al. What are cyclooxygenases and lipoxygenases doing in the driver's seat of carcinogenesis? Int J Cancer 2006;119: 2247-2254.

16 Chan G, Boyle JO, Yang EK, et al. Cyclooxygenase-2 expression is up-regulated in squamous cell carcinoma of the head and neck. Cancer Res 1999;59:991-994.

17 Zweifel BS, Davis TW, Ornberg RL, et al. Direct evidence for a role of cyclooxygenase 2-derived prostaglandin E2 in human head and neck xenograft tumors. Cancer Res 2002;62:6706-6711.

18 Williams CS, Tsujii M, Reese J, et al. Host cyclooxygenase-2 modulates carcinoma growth. J Clin Invest 2000;105:1589-1594.

19 Tsujii M, Kawano S, Tsuji S, et al. Cyclooxygenase regulates angiogenesis induced by colon cancer cells. Cell 1998;93:705-716.

20 Masferrer JL, Leahy KM, Koki AT, et al. Antiangiogenic and antitumor activities of cyclooxygenase-2 inhibitors. Cancer Res 2000;60:1306-1311.

21 Laimer K, Spizzo G, Obrist P, et al. STAT1 activation in squamous cell cancer of the oral cavity: a potential predictive marker of response to adjuvant chemotherapy. Cancer 2007;110:326-333.

22 Spizzo G, Gastl G, Wolf D, et al. Correlation of COX-2 and Ep-CAM overexpression in human invasive breast cancer and its impact on survival. Br J Cancer 2003;88: $574-578$. 
23 Gordon IO, Tretiakova MS, Noffsinger AE, et al. Prostate-specific membrane antigen expression in regeneration and repair. Mod Pathol 2008;21:1421-1427.

24 Wernicke AG, Edgar MA, Lavi E, et al. Prostate-specific membrane antigen as a potential novel vascular target for treatment of glioblastoma multiforme. Arch Pathol Lab Med 2011;135:1486-1489.

25 Zeng C, Ke ZF, Yang Z, et al. Prostate-specific membrane antigen: a new potential prognostic marker of osteosarcoma. Med Oncol 2011; e-pub ahead of print.

26 Chang SS, Reuter VE, Heston WD, et al. Metastatic renal cell carcinoma neovasculature expresses prostate-specific membrane antigen. Urology 2001;57: 801-805.

27 Gala JL, Loric S, Guiot Y, et al. Expression of prostatespecific membrane antigen in transitional cell carcinoma of the bladder: prognostic value? Clin Cancer Res 2000;6:4049-4054.

28 Conway RE, Petrovic N, Li Z, et al. Prostate-specific membrane antigen regulates angiogenesis by modulating integrin signal transduction. Mol Cell Biol 2006;26:5310-5324.

29 Yin L, Rao P, Elson P, et al. Role of TMPRSS2-ERG Gene Fusion in Negative Regulation of PSMA Expression. PLoS One 2011;6:e21319.

30 Evans MJ, Smith-Jones PM, Wongvipat J, et al. Noninvasive measurement of androgen receptor signaling with a positron-emitting radiopharmaceutical that targets prostate-specific membrane antigen. Proc Natl Acad Sci USA 2011;108:9578-9582.

31 Liu T, Jabbes M, Nedrow-Byers JR, et al. Detection of prostate-specific membrane antigen on HUVECs in response to breast tumor-conditioned medium. Int J Oncol 2011;38:1349-1355.
32 Hanahan D, Folkman J. Patterns and emerging mechanisms of the angiogenic switch during tumorigenesis. Cell 1996;86:353-364.

33 Folkman J. Angiogenesis. Annu Rev Med 2006;57:1-18.

34 Wang D, Dubois RN. Prostaglandins and cancer. Gut 2006;55:115-122.

35 Milowsky MI, Nanus DM, Kostakoglu L, et al. Vascular targeted therapy with anti-prostate-specific membrane antigen monoclonal antibody J591 in advanced solid tumors. J Clin Oncol 2007;25:540-547.

36 Morris MJ, Pandit-Taskar N, Divgi CR, et al. Phase I evaluation of J591 as a vascular targeting agent in progressive solid tumors. Clin Cancer Res 2007;13: 2707-2713.

37 Tagawa ST, Beltran H, Vallabhajosula S, et al. Antiprostate-specific membrane antigen-based radioimmunotherapy for prostate cancer. Cancer 2010;116: 1075-1083.

$38 \mathrm{Wu}$ P, Kudrolli TA, Chowdhury WH, et al. Adenovirus targeting to prostate-specific membrane antigen through virus-displayed, semirandom peptide library screening. Cancer Res 2010;70:9549-9553.

39 Lupold SE, Hicke BJ, Lin Y, et al. Identification and characterization of nuclease-stabilized RNA molecules that bind human prostate cancer cells via the prostatespecific membrane antigen. Cancer Res 2002;62: 4029-4033.

40 Chandran SS, Banerjee SR, Mease RC, et al. Characterization of a targeted nanoparticle functionalized with a urea-based inhibitor of prostate-specific membrane antigen (PSMA). Cancer Biol Ther 2008;7:974-982.

41 Elsasser-Beile U, Buhler P, Wolf P. Targeted therapies for prostate cancer against the prostate specific membrane antigen. Curr Drug Targets 2009;10:118-125. 